\title{
Successful focal ablation in a patient with electrical storm in the early postinfarction period: case report
}

This article was published in the following Dove Press journal:

International Medical Case Reports Journal

27 February 2015

Number of times this article has been viewed

\author{
Tolga Aksu' \\ Tumer Erdem Guler' \\ Ebru Golcuk ${ }^{2}$ \\ Kazım Serhan Ozcan' \\ Ismail Erden' \\ 'Department of Cardiology, Derince \\ Training and Research Hospital, \\ Kocaeli, Turkey; ${ }^{2}$ Department of \\ Cardiology, School of Medicine, Koç \\ University, Istanbul, Turkey
}

\begin{abstract}
Electrical storm (ES) is associated with a poor prognosis if it occurs in the early postinfarction period (within 4 weeks). There are limited data on the efficacy and safety of catheter ablation in the early period. In the patients with postinfarction cardiomyopathy, ventricular tachycardia (VT) is usually caused by re-entry through slowly conducting tissue within areas of a myocardial scar, whereas for the early postinfarction period, the underlying mechanism of ES is not fully understood. We report a case of ES for which macroreentry was excluded as a mechanism of VT because of the clinical and electrophysiological properties of the tachycardia. The tachycardia was terminated by focal radiofrequency catheter ablation of the earliest site. The total procedure time was only 35 minutes. During a 12-month follow-up period, the patient has remained free of monomorphic VT episodes. On the basis of this case, we aimed to discuss the underlying mechanism of ES in the early postinfarction period and to evaluate the role of radiofrequency catheter ablation as a primary approach for treating ES.
\end{abstract}

Keywords: ablation, electrical storm, ventricular tachycardia

\section{Learning objective}

In the early postinfarction period, the treatment of electrical storm (ES) can be challenging. The potential mechanism is usually macroreentry, and the ablation of an extensive area is usually necessary for success. However, macroreentry may be excluded simply by a detailed evaluation of the clinical and electrophysiological findings, and thus a permanent cure may be achieved by focal ablation.

\section{Introduction}

ES associated with ischemic cardiomyopathy (ICMP) is a rare clinical condition that is characterized by recurrent and intractable ventricular tachycardia (VT) and high mortality rates. ${ }^{1}$ Treatment options are antiarrhythmic drugs, antitachycardia pacing (ATP), electrical cardioversion, and, in refractory cases, deep sedation by general anaesthesia. ${ }^{2}$ The occurrence of ES during the late postinfarction period in patients with ICMP is usually scar-related, and macroreentry is usually the underlying mechanism. Ablation targets were determined by pace mapping during the sinus rhythm and by entrainment mapping during the sustained VT. ${ }^{3}$ Fractionated and isolated potentials and scars connected to adjacent nonconductive structures (mitral valve annulus) are additional ablation targets. On the other hand, focal VT related to ICMP is an uncommon arrhythmia, and the underlying mechanism has not been fully elucidated. Possible mechanisms include triggered activity, microreentry, abnormal automaticity, and transmural (epicardial-endocardial) re-entry.
Correspondence: Tolga Aksu

Department of Cardiology, Derince

Training and Research Hospital,

41900 Kocaeli, Turkey

Tel $+9053 \mid 9903278$

Fax +902623178000

Email aksutolga@gmail.com 
We report here the first ICMP case of ES for which focal application of radiofrequency catheter ablation (RFCA) was used as a primary approach to successfully terminate the tachycardia.

\section{Case report}

A 48 year-old male was referred to our clinic for refractory VT presenting as ES. The tachycardia was nonresponsive to administration of multiple intravenous antiarrhythmic drugs (1050 mg amiodarone, $150 \mathrm{mg}$ lidocaine, $15 \mathrm{mg}$ metoprolol succinate, and $2 \mathrm{~g}$ magnesium) and ATP. Multiple attempts of electrical cardioversion could not terminate VT. The last three cardioversion attempts resulted in the degeneration of VT to ventricular fibrillation. Repeated defibrillations with 200J, 200J, and 360J were performed, respectively, and VT continued.

In his medical history, he had undergone successful primary percutaneous coronary intervention for acute anterior myocardial infarction 10 days before this incident. He was admitted to the same center with sustained VT 3 days later, and control coronary angiography had revealed Thrombolysis In Myocardial Infarction 3 coronary flow in all coronary arteries.

His present admission electrocardiogram (ECG) was consistent with VT; the ECG showed a right bundle branch block morphology, south-west axis, and monophasic R wave in the augmented vector right (aVR, Figure 1). His admission blood pressure was 100/60 mmHg. No metabolic, acidbased, or electrolyte abnormalities were detected by serum biochemical parameters.

Transthoracic echocardiography revealed a left ventricular ejection fraction of $34 \%$ and moderate mitral regurgitation. Coronary angiography was repeated in our center. Hemodynamically significant stenosis was not detected (Figure 2), and acute ischemia was excluded as a cause of VT.

Electrophysiological study was performed under local anesthesia and conscious sedation. The ECG findings were consistent with VT originating from the left ventricle (LV). A $4 \mathrm{~mm}$ quadripolar diagnostic catheter was applied to right atrium/right ventricle through the right femoral vein. A $3.5 \mathrm{~mm}$ open irrigated tip ablation catheter (Thermocool; Biosense-Webster, Inc., Diamond Bar, CA, USA) was used for the mapping and the ablation of the LV by using a retrograde aortic approach. Electrophysiology study revealed atrioventricular dissociation and confirmed that the VT originated from the LV (Figure 3).

A 9F Ensite Array noncontact mapping catheter (St Jude Medical, St Paul, MN, USA) was positioned in the LV.

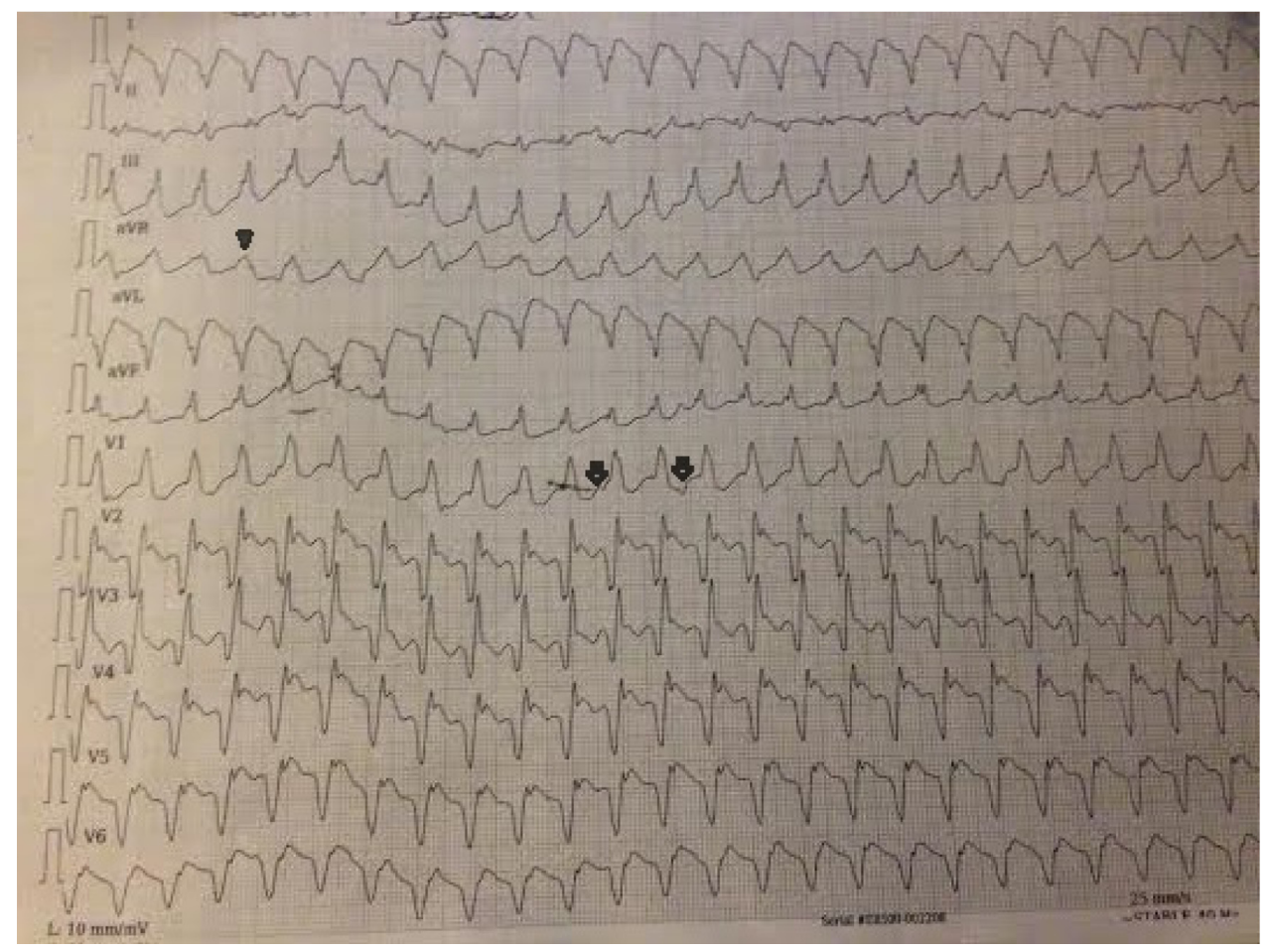

Figure I The patient's ECG upon admission.

Notes: Twelve-lead ECG upon admission revealed wide QRS tachycardia with RBBB morphology, south-west axis, monophasic R wave in aVR (arrow-head), and atrioventricular dissociation (arrow).

Abbreviations: ECG, electrocardiogram; RBBB, right bundle branch block; aVR, augmented vector right. 


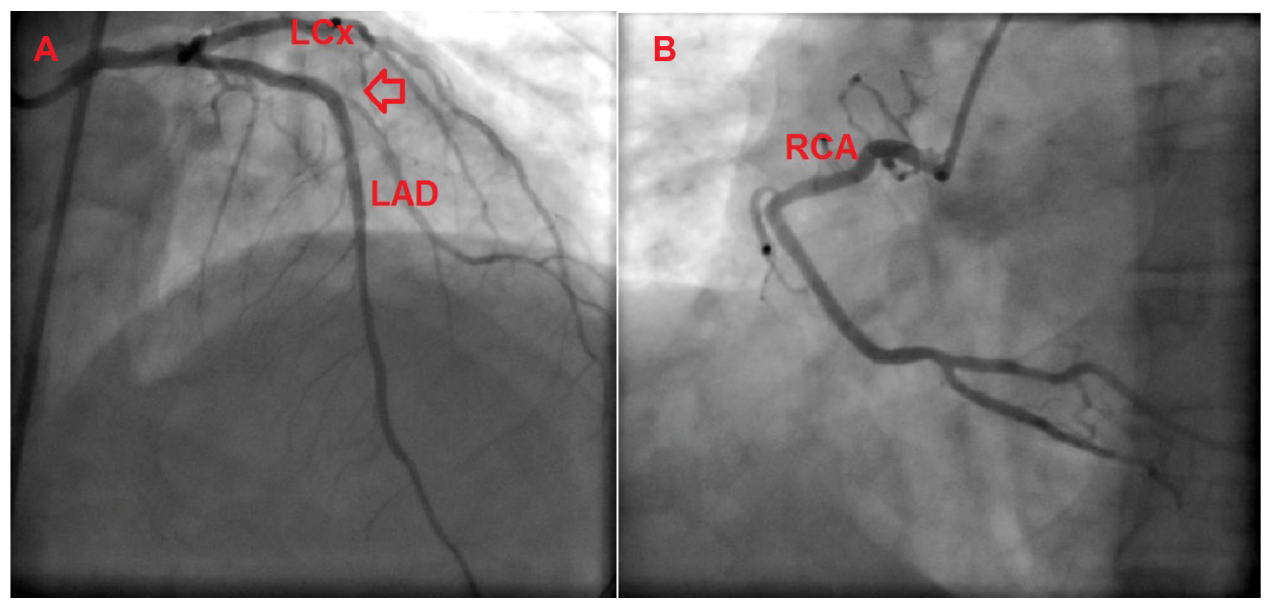

Figure 2 Coronary angiographic views of the patient.

Notes: Coronary angiogram shows left anterior descending artery and left circumflex artery; the arrow demonstrates an implanted coronary stent (A). Coronary angiogram shows right coronary artery (B).

Abbreviations: LAD, left anterior descending artery; LCx, left circumflex artery; RCA, right coronary artery.

Then, the activation mapping of the LV was performed by using the ablation catheter. The earliest local ventricular point was localized to the lateral wall of the LV apex (Figure 4A).

Activation mapping revealed a consistent and centrifugal pattern of activation during the tachycardia (Movie 1). Also, there was an absence of substantial conduction delay in the vicinity of the focus of earliest activity and hence made macroreentry unlikely. So, increased automaticity, microreentry, and triggered activity were considered as three potential causative mechanism of focal-originating VT. Under these circumstances, microreentry could not be entirely excluded, although we have speculated that mechanisms such as triggering or automaticity might be more likely because the tachycardia had not been responsive to overdrive pacing and

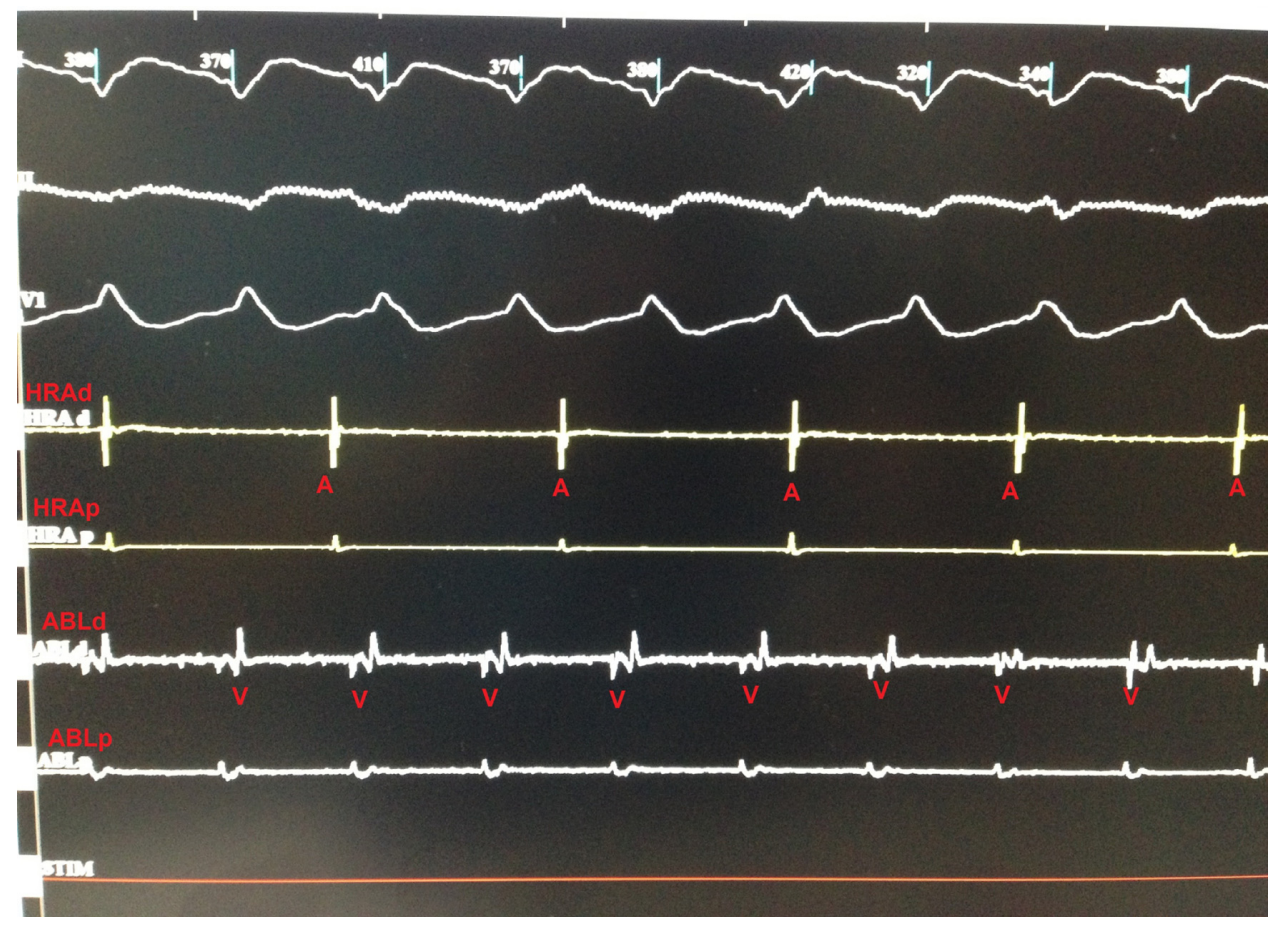

Figure 3 Tracing from the electrophysiology study showing ventricular tachycardia intracardiac electrogram during ventricular tachycardia.

Notes: I, II and VI were surface electrocardiography leads. HRA and ABL are intracardiac leads (HRA is the diagnostic catheter and placed in high right atrium, ABL is the ablation catheter and placed in the left ventricle).

Abbreviations: HRA, high right atrium; $A B L$, ablation catheter; $A$, atrium; $V$, ventricle; $d$, distal; p, proximal. 


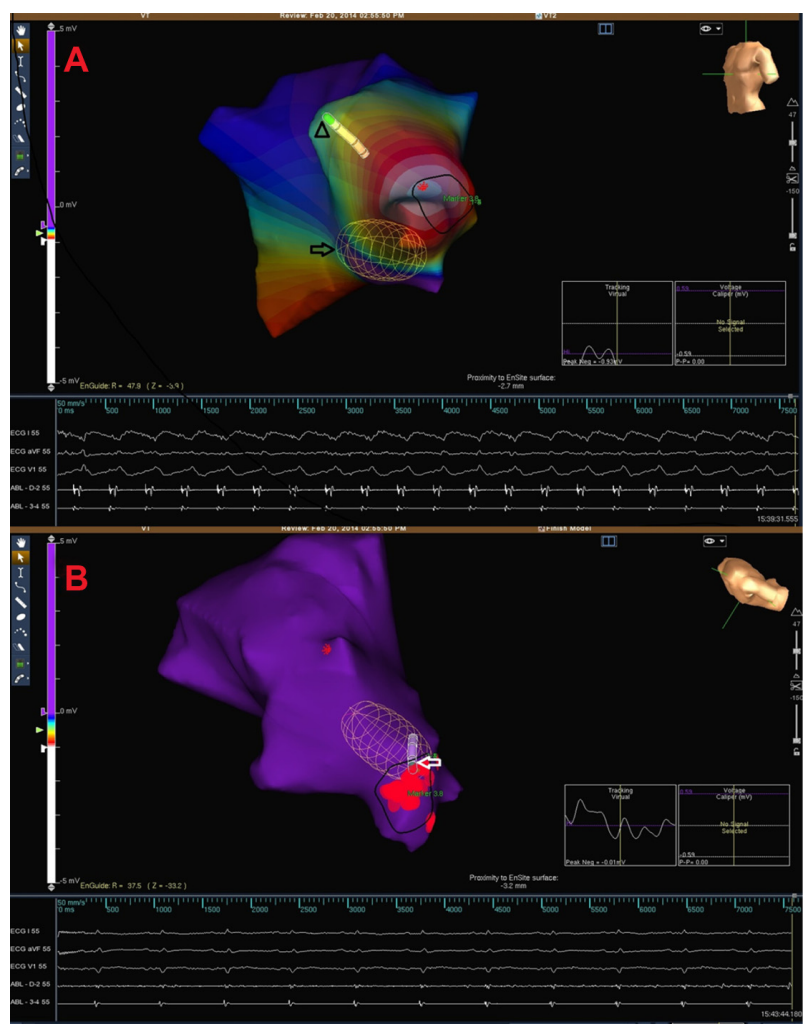

Figure 4 Electrocardiograms and endocardial activation maps.

Notes: Electrocardiograms and endocardial activation map of ventricular tachycardia. Arrow indicates the Ensite Array catheter. The ablation catheter (arrowhead) is seen as shaded (A). Electrocardiograms and endocardial activation map after successful ablation. The ablation catheter (arrow) is in the region of sinus rhythm is achieved (B).

degenerated to ventricular fibrillation after ATP. In fact, it is well known that entrainment occurs because of a specific response of tachycardia to overdrive pacing, and entrainment of a tachycardia can be a useful method to identify re-entry as a mechanism.

Because the tachycardia was considered as a focaloriginating VT, we chose to perform focal ablation of the earliest site without scar mapping to shorten the procedure. The earliest site was ablated with $35 \mathrm{~W}, 40$ degrees, and VT was terminated after exactly 30 seconds of radiofrequency application (Figure 4B). We applied ablation to the surrounding area to increase the efficiency of ablation. Clinical tachycardia was not induced either by the programmed stimuli or an isoproterenol infusion of up to $20 \mu \mathrm{g} / \mathrm{min}$ after the procedure. The total duration of the procedure was 35 minutes, and total fluoroscopy time was only 9 minutes. Two days later, a single-chamber defibrillator (ICD) was implanted, and the patient was discharged with beta blocker treatment. The patient was asymptomatic at the 6-month and 1-year follow-ups. Defibrillator records did not reveal any ventricular arrhythmia episodes.

\section{Discussion}

ES occurring after 24 hours but within 4 weeks of acute myocardial infarction is clinically rare, and the mortality rate with antiarrhythmic drugs is over $50 \% .{ }^{1}$ High mortality rates are related with direct cell injury by frequent delivery of intracardiac defibrillator shocks or with renal and hepatic function impairments caused by low cardiac output for a long period of time. ${ }^{4}$ There are a lot of studies about RFCA for VT in the late postinfarction period. However, for the early postinfarction period, the underlying mechanism of tachycardia and the safety and efficacy of RFCA are still not well known.

Contrary to our case, ES occurring in the early postinfarction period is often attributed to acute ischemia or electrolyte abnormalities. A macroreentrant mechanism is the most common arrhythmia mechanism in a postinfarct substrate; it is particularly difficult to exclude the possibility of triggering or automaticity in our case. To exclude macroreentry from the other potential mechanisms, some confusing electrophysiological characteristics should be kept in mind. Firstly, noncontact mapping based on virtual unipolar electrograms has limitations in identifying an activation wavefront within the scar because of intrinsically low $\mathrm{dV} / \mathrm{dT}$ in these regions. Secondly, the absence of response to overdrive pacing usually suggests that macroreentry is not likely to be the underlying mechanism, but this factor does not exclude macroreentry entirely, although a consistent response to entrainment may confirm re-entry. The ability to terminate sustained VT by overdrive pacing is influenced by the refractoriness at the stimulation site, the conduction time from the stimulation site to the tachycardia origin, the tachycardia cycle length, and the duration of the excitable gap. ${ }^{5}$ Lastly, a transmural (epicardialendocardial) re-entry may exhibit a focal endocardial exit point with centrifugal activation and a lack of classical earlymeets-late-activation wavefronts; when this happens, often a focal mechanism is simulated as a result.

Radiofrequency ablation of postinfarction VT is often complex because of the necessity for repeated induction attempts and prolonged mapping of sustained VT. When possible, 12-lead ECGs of the clinical VT should be obtained. The morphology of the VT may help to localize the exit site of the re-entrant circuit from the protected isthmus. The morphology of right bundle branch block in lead V1 identifies a LV exit site, whereas a left bundle branch block morphology suggests a VT exit in the right ventricle or, more commonly, the LV septum. More specific localization can be achieved by using the aVR and the augmented vector left (aVL) leads to distinguish septal from lateral exit, by using leads II and III 
and the augmented vector foot (aVF) lead to distinguish superior from inferior exit, and by using the precordial leads to identify an apical/mid/basal exit.

Postinfarction VT is caused by re-entry through diseased myocardium due to prior myocardial infarction. These scar areas consist of fibrotic, unexcitable tissue interspersed with areas of surviving myocytes and areas of functional block that lead to slow conduction and unidirectional block critical to initiating and maintaining re-entry.

If VT is sustained, the recording of the earliest activation site can be achieved by using an electroanatomical mapping system showing an activation map. Entrainment mapping is then performed near the site of the earliest activation. The response to pacing helps to identify the site of the re-entrant circuit as entrance, isthmus, exit, inner loop, outer loop, adjacent bystander, or remote bystander components. After entrainment mapping, RFCA of the critical isthmus site is the first ablation target. However, the procedures that were previously described are rather complex, and the procedure time may be as long as 5 hours. ${ }^{6}$

When we evaluated both the clinical and electrophysiological findings on our patient, the tachycardia was considered to be of focal origin. The success of focal ablation in a short time was due to our foresight.

Carbucicchio et $\mathrm{al}^{7}$ prospectively evaluated 95 patients who underwent RFCA for ES; 76\% of these patients had coronary artery disease. At the end of the 22 month followup, the recurrence rate of ES was $8 \%$.

Despite successful termination of tachycardia episodes, ICDs have no effect on the arrhythmic substrate, which is usually progressive. In addition, it has been shown that delivery of shocks is associated with impaired quality of life and increased mortality. ${ }^{8}$ Currently, however, the implantation of the ICD is the only treatment method that has been shown to reduce the mortality in the patients with ICMP who present with VT. ${ }^{9}$ So, the successful ablation of clinical VT should not be thought of as the clinical end point.

\section{Conclusion}

We conclude that if macroreentry can be excluded as a mechanism of the tachycardia on the basis of clinical and electrophysiological findings, the ablation of a restricted area is a safe and effective treatment option in an experienced referral center. This type of ablation is also associated with a decreased risk of complications.

\section{Disclosure}

The authors declare no conflicts of interest in this work.

\section{References}

1. Exner DV, Pinski SL, Wyse G, et al; AVID Investigators. Electrical storm presages nonsudden death: the antiarrhythmics versus implantable defibrillators (AVID) trial. Circulation. 2001;103(16):2066-2071.

2. Kowey PR. An overview of antiarrhythmic drug management of electrical storm. Can J Cardiol. 1996;12(Suppl B):3B-8B; discussion 27B-28B.

3. Wissner E, Stevenson WG, Kuck KH. Catheter ablation of ventricular tachycardia in ischaemic and non-ischaemic cardiomyopathy: where are we today? A clinical review. Eur Heart J. 2012;33(12):1440-1450.

4. Wilson CM, Allen JD, Bridges JB, Adgey AA. Death and damage caused by multiple direct current shocks: studies in an animal model. Eur Heart J. 1988;9(11):1257-1265.

5. Josephson ME. Clinical Cardiac Electrophysiology: Techniques and Interpretations. 4th ed. Philadelphia: Lippincott Williams and Wilkins; 2008.

6. Reddy VY, Neuzil P, Taborsky M, Ruskin JN. Short-term results of substrate mapping and radiofrequency ablation of ischemic ventricular tachycardia using a saline-irrigated catheter. $J$ Am Coll Cardiol. 2003;41(12):2228-2236.

7. Carbucicchio C, Santamaria M, Trevisi N, et al. Catheter ablation for the treatment of electrical storm in patients with implantable cardioverterdefibrillators: short- and long-term outcomes in a prospective singlecenter study. Circulation. 2008;117(4):462-469.

8. Powell BD, Saxon LA, Boehmer JP, et al. Survival after shock therapy in implantable cardioverter-defibrillator and cardiac resynchronization therapy-defibrillator recipients according to rhythm shocked. The ALTITUDE survival by rhythm study. J Am Coll Cardiol. 2013;62(18): 1674-1679.

9. Bardy GH, Lee KL, Mark DB, et al; Sudden Cardiac Death in Heart Failure Trial Investigators. Amiodarone or an implantable cardioverterdefibrillator for congestive heart failure. $N$ Engl J Med. 2005;352(3): 225-237.
International Medical Case Reports Journal

\section{Publish your work in this journal}

The International Medical Case Reports Journal is an international, peer-reviewed open-access journal publishing original case reports from all medical specialties. Previously unpublished medical posters are also accepted relating to any area of clinical or preclinical science. Submissions should not normally exceed 2,000 words or

\section{Dovepress}

4 published pages including figures, diagrams and references. The manuscript management system is completely online and includes a very quick and fair peer-review system, which is all easy to use. Visit $\mathrm{http}: / / \mathrm{ww} w$.dovepress.com/testimonials.php to read real quotes from published authors. 